\title{
ОПРЕДЕЛЕНИЕ РАЗМЕРОВ СТРУКТУРНЫХ ЭЛЕМЕНТОВ НА ПОВЕРХНОСТИ КОНДЕНСИРОВАННЫХ СРЕД ПУТЕМ ВЕЙВЛЕТ-ПРЕОБРАЗОВАНИЯ СГЕНЕРИРОВАННЫХ ОПТИЧЕСКИХ ИЗОБРАЖЕНИЙ
}

\author{
(C) 2018 М. М. Филяк, А. Г. Четверикова, О.Н.Каныгина \\ Оренбургский государственный университет, пр-т Победы, 13, 460018 Оренбург, Россия \\ e-mail:KR-727@mail.ru
}

Поступила в редакцию 26.01.2018

\begin{abstract}
Аннотация. Предложен метод определения размеров структурных элементов на поверхности конденсированных сред путем моделирования их изображений и применения вейвлет-преобразований. Параметр масштаба вейвлет-спектров модельных изображений позволяет оценивать размеры частиц в реальных мономорфных дисперсных системах. Показано на примере гомогенных глинистых порошков, что в микрометровой области погрешность определения эквивалентных диаметров частиц не превышает $\pm 10 \%$.
\end{abstract}

Ключевые слова: поверхность конденсированной среды, структурный элемент, размер, изображение, модель, вейвлет-спектр.

DOI: https://doi.org/10.17308/kcmf.2018.20/487

\section{ВВЕДЕНИЕ}

Изучение структуры и состава поверхности одна из наиболее важных проблем физики поверхности, успехи в области которой обуславливают достижения в современной микро- и наноэлектронике, гетерогенном катализе, космических технологиях $[1,2]$.

Основной задачей обработки изображений поверхностей конденсированных сред является извлечение характерных информативных признаков присутствующих на нем объектов. Выделение структурных элементов в субмикронных, микронных, миллиметровых масштабах на изображениях поверхностей конденсированных сред является сложной задачей для дисперсионного и морфологического анализов $[3,4]$. Первостепенное значение в таких случаях отводится определению размеров и форм частиц в дисперсных системах, размеров зерен в поликристаллах, ячеек и пор в порошковых материалах, поскольку эти параметры определяют многие функциональные свойства твердых тел. Особый интерес в подобных исследованиях, несмотря на развитие инструментальной и мето- дической баз, по-прежнему представляют прямые методы обработки микроскопических изображений поверхностей конденсированных сред.

Исследование поверхностей конденсированных сред заключается в обработке и анализе информации о периодичности, форме и размерах структурных элементов, характере их распределения в двумерных координатах. Способ выделения информации из изображения является ключевой проблемой, для решения которой необходимо применение компьютерных технологий, позволяющих охарактеризовать не только отдельные элементы, но и свойства объекта в целом $[2,5]$. В последнее время подобные структурные исследования проводятся с использованием непрерывного вейвлет-преобразования. Успешные результаты такого подхода изложены в работах $[6,7]$.

Известны примеры применения метода вейвлет-преобразования для решения задач, связанных с экспериментальным исследованием структуры поверхностей материалов, в частности, для определения шероховатости поверхности [8], структуры титановых губок [9], толщины и угла смачивания 
порошково-обжигового покрытия [10]. Авторами на основе вейвлет-анализа цифровых изображений поверхностей проведены исследования эволюции морфологии дисперсных частиц глинистых минералов при микроволновом воздействии [11].

Поверхности современных конденсированных сред часто имеют сложные структуры, дающие слабоконтрастные изображения, улучшение которых приводит, как правило, к искажению информации. Авторами работы [7] рассмотрен новый подход к решению практической проблемы - оценке качества сварных швов (наблюдения локальных дефектов) путем фильтрации полезного сигнала, выделения объектов на низкоконтрастных изображениях, генерации модельных изображений и обработки их непрерывным вейвлет-преобразованием.

Вейвлеты представляют собой особые функции в виде коротких волн с нулевым интегральным значением и локализацией по оси времени (координат), способных к сдвигу по этой оси и масштабированию (растяжению/сжатию). Подобное преобразование можно применять как к временным зависимостям, так и к пространственным распределениям [12]. Вейвлет-спектры обеспечивают двумерную развертку исследуемого сигнала, определение его локальных и интегральных характеристик и возможность анализировать свойства сигнала одновременно в физическом (время, координата) и в частотном пространствах.

В работе предложен метод определения размеров структурных элементов поверхности конденсированных сред на основе вейвлет-преобразования сгенерированных моделей. Выбор метода обусловлен возможностью перехода к частотно-пространственному представлению без предварительной обработки изображений. Такой подход, обеспечивающий выделение объектов путем фильтрации полезного сигнала, предпочтительней традиционных количественных характеристик объектов на изображениях за счет большой статистической выборки и способности адаптации к практически неограниченному количеству морфологических особенностей, наблюдаемых для конденсированных сред.

\section{ЭКСПЕРИМЕНТАЛЬНАЯ ЧАСТЬ}

Вейвлет-анализ поверхностей конденсированных сред представляет собой обработку изображений с использованием современного математического аппарата и компьютерных технологий. Изображение на экране монитора микроскопа формируется в виде матрицы из $n$ строк по $m$ пикселей в каждой, яркость которых прямо пропорциональна значению сигнала соответствующей точки матрицы. Прежде чем приступить к определению размеров частиц необходимо установить их форму. В простейшем случае частицам присваивается сферическая форма, однако сферические частицы встречаются в особых случаях. Все частицы в стереологии по форме делят на изометрические, пластинчатые и волокна. Для характеристики формы частиц неправильной формы часто используют фактор формы или несферичность, а для оценки размеров - понятие эквивалентного диаметра. Реальные частицы (преимущественно изометрической или осколочной формы) заменяют на эквивалентные частицы правильной геометрической формы (например, прямоугольники или тетраэдры) с использованием среднего проектированного диаметpa, площадь которого равна площади изображения проекции частицы [13].

Авторами работы в качестве эталонных объектов для моделирования выбраны правильные тетраэдры, при этом диаметры сфер, вписанных в тетраэдр, соответствуют эквивалентным диаметрам частиц.

Модельные изображения получали компиляцией тетраэдров заданных размеров при помощи компьютерной программы Gwyddion. На рис. 1 показаны изображения сгенерированных поверхностей с размерами частиц 240 (рис. 1 ) и 600 (рис. 1 ) мкм. Эти значения - верхние стандартные показатели при гранулометрическом анализе природных диспергированных систем.

На первом этапе определения количественных характеристик структурных элементов получали профили рельефа исследуемой поверхности. В данном случае они представляют собой зависимости «яркости» точек цифрового изображения от пространственной координаты, полученные с помощью программы ImageJ. Вейвлет-анализ полученных профилей проводили, используя программy Spectra Analyzer, как и авторы [2] на основе Morlet-вейвлета.

Вейвлет-преобразование произвольной функции $z(x)$ определяется как интеграл вида:

$$
W(m, n)=\frac{1}{\sqrt{m}} \int_{-\infty}^{+\infty} z(x) \cdot \psi\left(\frac{x-n}{m}\right) d x .
$$

Результатом вейвлет-преобразования является вейвлет-спектр $W(m, n)$ - функция двух переменных: параметра масштаба вейвлета $-m$ и параметра сдвига вейвлета $-n$.

Выбор базисного вейвлета $\Psi(x)$ определяется характером информации, извлекаемой из сигна- 


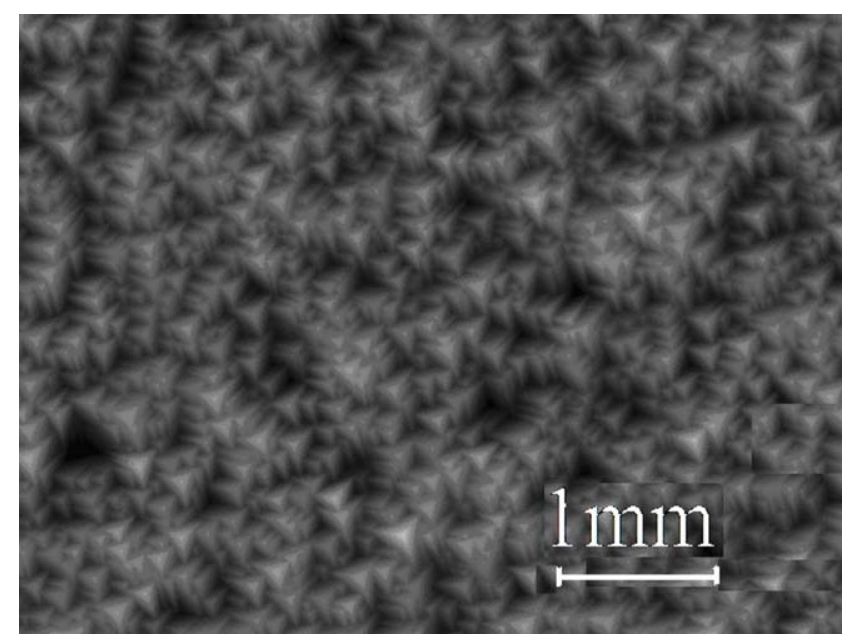

$a$

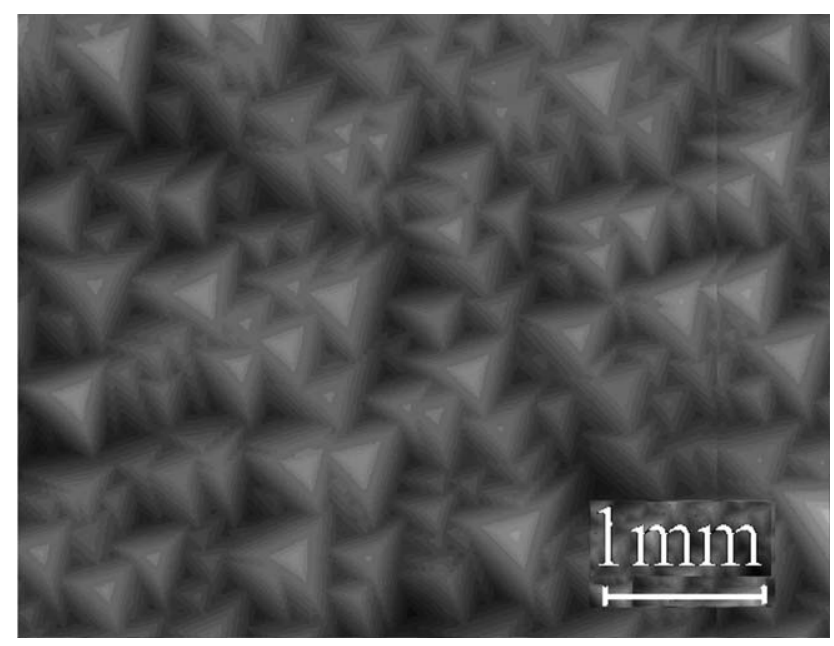

$b$

Рис. 1. Изображения смоделированных поверхностей с размерами частиц (мкм): $240(a)$ и $600(b)$

[Fig. 1. Images of simulated surfaces with particle sizes $(\mu \mathrm{m}): 240(a)$ and $600(b)]$

ла [5]. Сравнительный анализ различных вейвлетбазисов показал, что наиболее точно и полно выявить особенности профиля физических поверхностей позволяет Morlet-вейвлет, описываемый выражением [14]:

$$
\psi(x)=e^{\text {const } x} \cdot e^{-x^{2} / 2} .
$$

Morlet-вейвлет обеспечивает наилучшее соотношение между разрешением по координате и частоте.

\section{РЕЗУЛЬТАТЫ И ИХ ОБСУЖДЕНИЕ}

На рис. 2 приведены вейвлет-спектры для двух модельных образцов, где по оси абсцисс отложен параметр сдвига вейвлета $n$ (вдоль изображения), измеряемый в пикселях; по оси ординат - параметр масштабирования базисного вейвлета т. Профиль любой физической поверхности можно представить как ряд пространственных частот. В таком случае нижняя часть спектрограммы соответствует малым значениям масштаба и представляет детальную картину рельефа. Эти «высокочастотные» неоднородности являются отражением мелких структурных элементов. В центральной части вейвлет-спектра расположены компоненты «средней частоты», ответственные за наличие и распределение более крупных структурных элементов, а вверху - большие «низкочастотные» значения, дающие сглаженный рельеф поверхности.

Величины коэффициентов вейвлет-преобразования в точке тем больше, чем лучше корреляция между вейвлет-базисиом данного масштаба $m$ и профилем поверхности в ее окрестности $[12,14]$. Цвет вейвлет-спектра определяется величиной ко- эффициентов: белый цвет отвечает за максимальное, черный - за минимальное значение. Различие в цветах спектрограммы свидетельствует о наличии структурных элементов разной величины.

Для образца со средним размером структурных элементов 240 мкм корреляция между рельефом поверхности и базовым вейвлетом отсутствует вплоть до значений $m=2$ (сине-фиолетовые участки на рис. $2 a$ ). На изображении поверхности, смоделированной из частиц с эквивалентным диаметром, равным 600 мкм, нет корреляции вплоть до $m=3$. Смещение темных участков по вертикали на единицу вверх подтверждает, что на рис. $2 b$ показан больший размер частиц.

По цвету областей спектрограмм, принадлежащих разным масштабам, можно не только судить о наличии частиц с разными размерами, но и определить эти размеры.

На обоих вейвлет-спектрах отчетливо видны повторяющиеся однотипные элементы - так называемые «арки» $[6,15]$ с зелеными границами и сине-фиолетовой серединой. Размеры частиц можно определить, измеряя «высоту» $m$, на которой располагаются вершины этих арок. На спектрограмме образца с размерами частиц 240 мкм вершины арок располагаются на значениях масштаба 3.0-3.1, а на спектрограмме образца с размерами частиц 600 мкм они смещаются до значений масштаба 3.8-4.0.

В результате проведенных расчетов установлено, что размер частиц связан с порядком масштаба m, на котором находятся вершины арок простым соотношением: 
m, усл.ед.

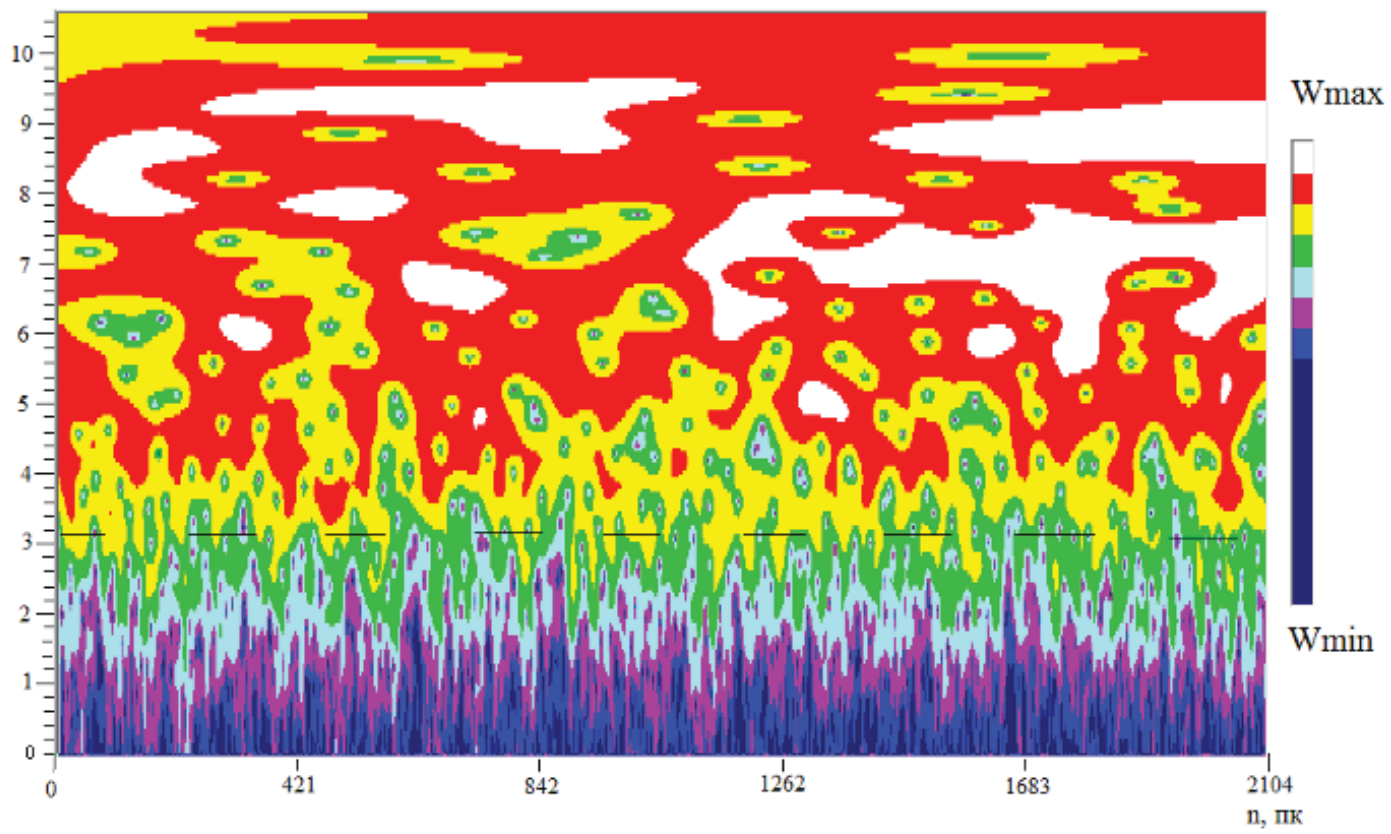

$a$

m, усл.ед.

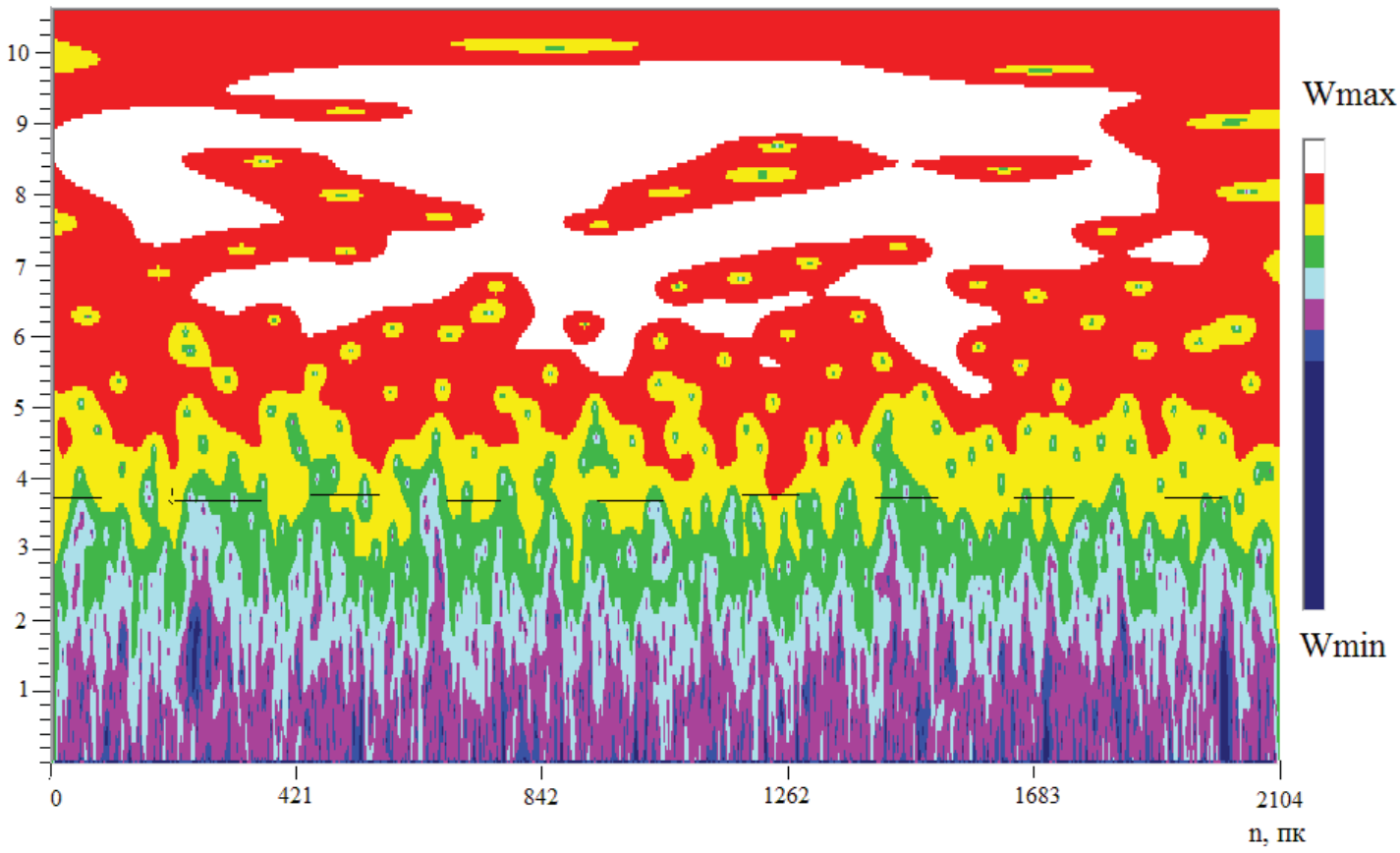

$b$

Рис. 2. Вейвлет-спектры смоделированных поверхностей с размерами частиц (мкм): $240(a)$ и $600(b)$

[Fig. 2. Wavelet spectra of simulated surfaces with particle sizes $(\mu \mathrm{m})$ : $240(a)$ and $600(b)]$ 


$$
m=\ln r_{\text {пाк }},
$$

где $r_{\text {пк }}$ - размер частиц в пикселях.

Размер частиц в микрометрах определяется по соотношению:

$$
R_{\text {мкм }}=K \cdot r_{\text {пк' }}
$$

где значение $K$ определяется увеличением и разрешением изображения.

В нашем случае $K=12$, следовательно, размеры частиц лежат в диапазоне (мкм) $240 \pm 10$ и $590 \pm 30$, совпадая с заданными размерами структурных элементов в сгенерированных моделях.

На практике монодисперсные системы встречаются редко, поэтому представляет интерес проверить эффективность нового подхода к определению размеров частиц в полидисперсной системе. На рис. $3 a$ представлено изображение сгене- рированной модели с размерами частиц 120, 240 и 600 мкм (слева направо), разделенных границами, а на рис. $3 b$ - вейвлет-спектр модельного образца.

Вейвлет-спектр отражает наличие в модели трех областей с разной высотой арок и позволяет выявить частицы разных размеров, а также их локализацию. Смещение границ арок вверх при переходе из левой части в правую на рис. $3 b$ показывает, что размеры частиц увеличиваются. Расчет эквивалентных диаметров частиц с использованием соотношений (1) и (2) дает следующие результаты (мкм): $120 \pm 10,240 \pm 10,600 \pm 20$.

Метод вейвлет-преобразования проверен на порошках природной глины, содержащих частицы осколочной формы, после рассева на ситах с ячейками 630 и 600 мкм $(600 \leq d \leq 630$ мкм). На рис. 4 представлено изображение поверхности порошка глины

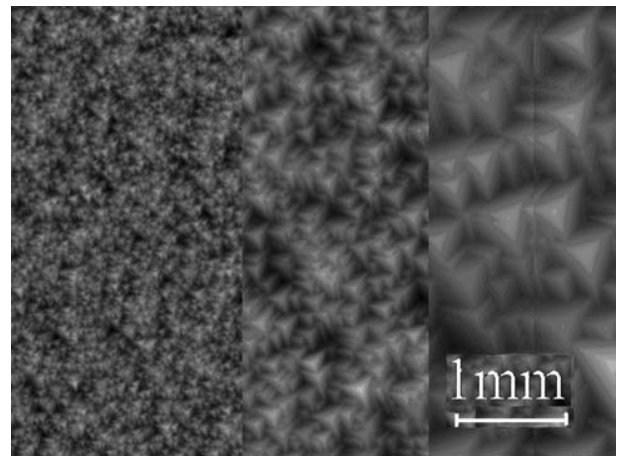

$a$

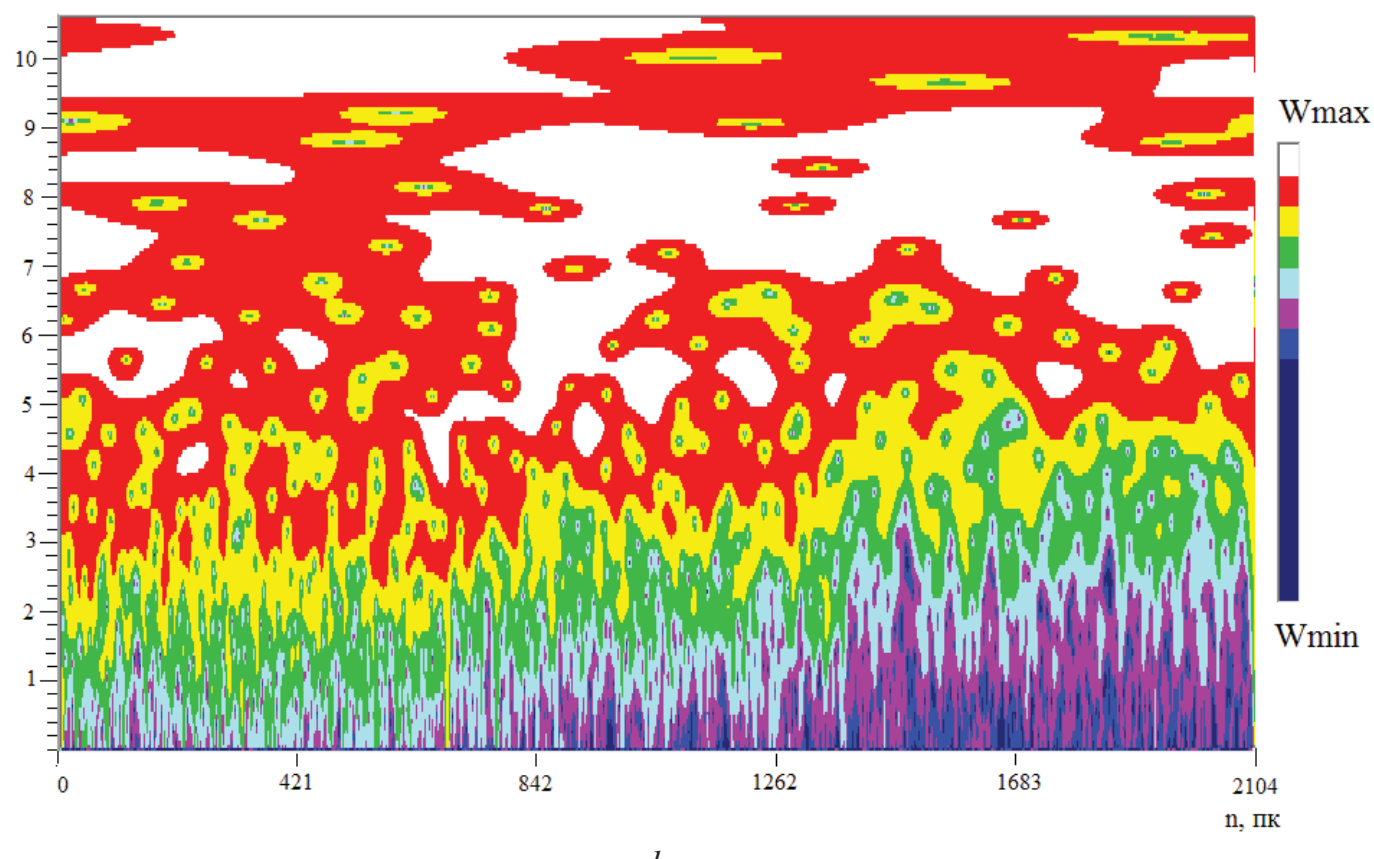

$b$

Рис. 3. Изображение (a) и вейвлет-спектр $(b)$ смоделированных поверхностей с частицами размером (мкм): 120 , 240 и 600

[Fig. 3. Image $(a)$ and wavelet spectrum $(b)$ of simulated surfaces with particles of size $(\mu \mathrm{m}): 120,240$ and 600 ] 


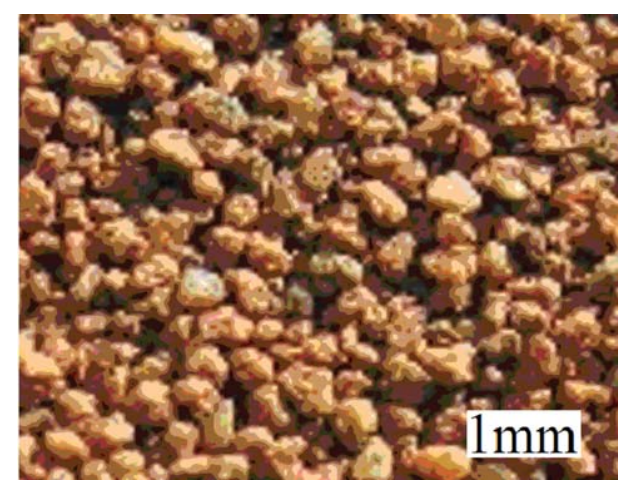

$a$

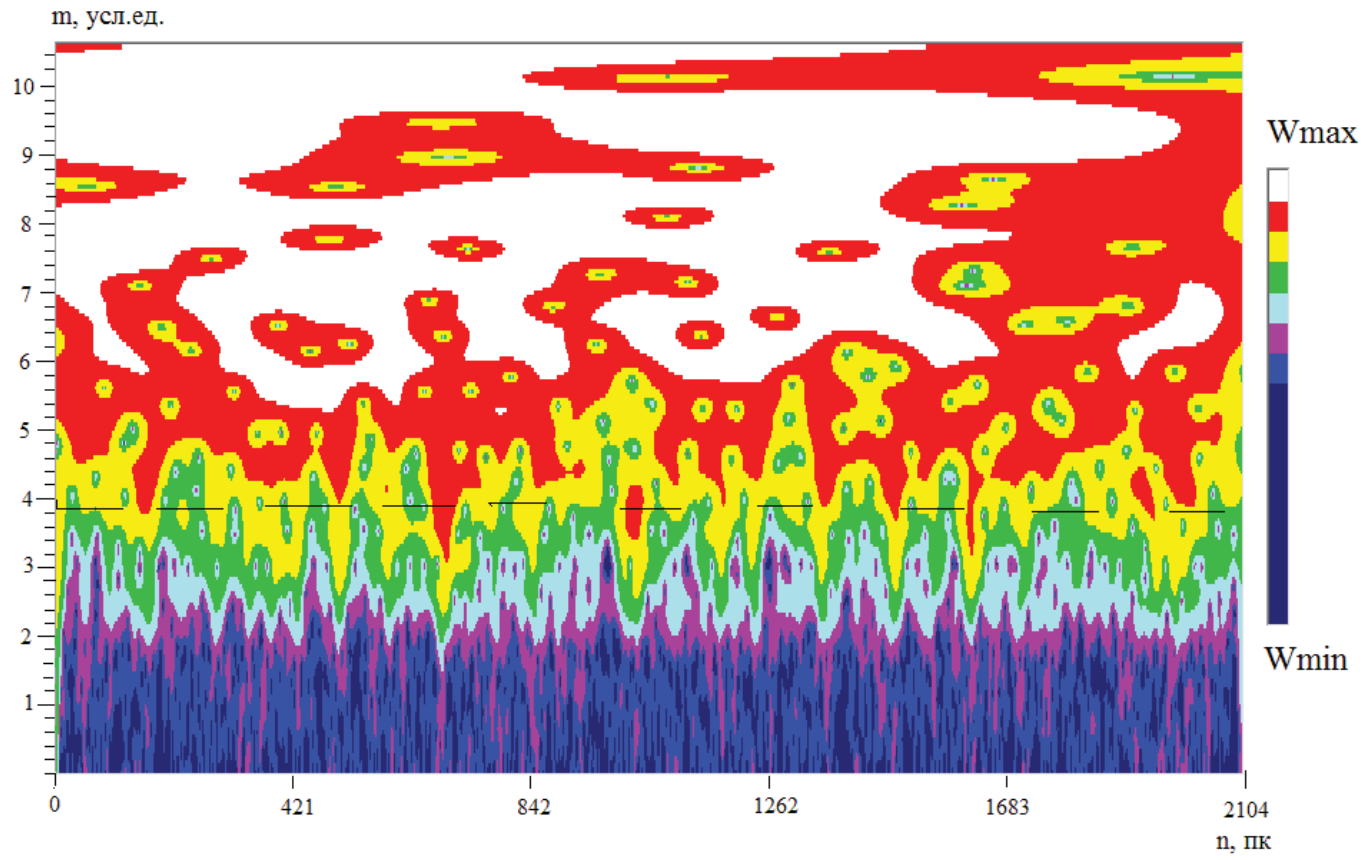

$b$

Рис. 4. Изображение поверхности порошка глины с размерами частиц $600 \leq d \leq 630$ мкм ( $a$ ), его вейвлет-спектр $(b)$

[Fig. 4. The image of the surface of a clay powder with particle sizes $600 \leq d \leq 630 \mu \mathrm{m}(a)$, its wavelet spectrum $(b)$ ]

и его вейвлет-спектр. Частицы (рис. $4 a$ ) имеют преимущественно осколочную форму, близкую к изометрической (тетраэдрической). На вейвлет-спектре (рис. 4b) пунктирной линией отмечены арки, высота которых соответствует значениям масштаба $m=3.9$ 4.0. Поверхность образца имеет квазипериодическое строение с шагом 50-55 пкс (620 20 мкм), что совпадает с эквивалентными диаметрами частиц, определенными ситовым анализом.

\section{ЗАКЛЮЧЕНИЕ}

Показана целесообразность применения непрерывного вейвлет-преобразования для оценки размеров элементов структуры поверхности конденсированных сред по сгенерированным модельным изображениям. Метод позволяет оценивать значе- ния эквивалентных диаметров частиц неправильной формы в реальных мономорфных системах, выявлять неоднородности и структурные особенности. Удовлетворительная информативность и достоверность метода обусловлена значительной статистической выборкой измерений. В субмиллиметровой и микрометровой области погрешность определения значения эквивалентных диаметров частиц в дисперсных системах не превышает $\pm 10 \%$.

Предлагаемый подход к исследованию фазовых морфологий в конденсированных средах дополняет перечень методов исследования поверхностных структур реальных материалов.

Работа выполнена при финансовой поддержке РФФИ и правительства Оренбургской области в 
рамках научного проекта №17-42-560069 p_a «Новые оптико-математические методы анализа структурных вариаций дисперсных и наноструктурированных систем».

\section{СПИСОК ЛИТЕРАТУРЫ}

1. Шкатов В. В., Шатов Ю. С., Щеренкова И. С. // Конденсированные среды и межфазные границы, 2013, т. 15, № 2, с. 195-199.

2. Постников Е. В., Лоскутов А. Ю. // Журнал экспериментальной и теоретической физики, 2007, т. 131, № 3, c. 466-471. DOI: 10.1134/S1063776107030065

3. Mel'nik V. M., Rud' V. D. \& Mel'nik Y. A. // Powder Metallurgy and Metal Ceramics, 2014, vol. 53, № 1-2, pp. 107-112.

4. Филяк М. М., Четверикова А. Г., Каныгина О. Н., Багдасарян Л. С. // Конденсированные среды и межфазные границы, 2016, т. 18, № 4, с. 578-585.

5. Колмаков А. Г., Солнцев К. А., Витязь П. А., Ильющенко А. Ф., Хейфец М. Л., Баринов С. М. // Материаловедение, 2012, № 9, с. 37-45. DOI: 10.1134/ S2075113313040059

6. Машкина Е. С., Гречкина М. В. // Конденсированные среды и межфазные границы, 2013, т. 15, № 1, c. $28-33$.

7. Жизняков А. Л., Фомин А. А. // Фундаментальные проблемы современного материаловедения, 2007, т. 4, № 2 , с. $75-80$.
8. Добротворский С. С., Басова Е. В., Репета А. А. // Вестник национального технического университета Харьковский политехнический институт. Серия: информатика и моделирование, 2011, № 13, с. 75-82.

9. Зверев В. В., Залазинский А. Г., Новоженов В. И, Поляков А. П. // Прикладная механика и техническая физика, 2001, т. 42, № 9, с. 199-207. DOI: https://doi. org/10.1023/A:1018856726111

10. Махов В. Е. // Конструкциии из композиционных материалов, 2010, № 3, с. 28-36.

11. Четверикова А. Г., Каныгина О. Н., Филяк М. М., Савинкова Е. С. // Измерительная техника, 2017, № 11, c. $27-31$.

12. Гиляров В. Л., Корсунков В. Е., Бутенко П. Н., Светлов В. Н. // ФТT, 2004, т. 46, № 10, с. 1806-1810. DOI: https://doi.org/10.1134/1.1809422

13. ГОСТ Р 8.755-2011 Государственная система обеспечения единства измерений. Дисперсный состав газовых сред. Определение размеров наночастии методом диффузионной спектрометрии. М.: Стандартинформ, 2013, $30 \mathrm{c}$.

14. Астафьева Н. М. // Успехи физических наук, 1996, т. 166, № 11, c. 1145-1150. DOI: http://dx.doi.org/10.1070/ PU1996v039n11ABEH000177

15. Filyak M. M., Chetverikova A. G., Kanygina O. N., Savinkova E. S., Berdinskiy V. L. // Proceedings of the 14th Sino-Russia Symposium on Advanced Materials and Technologies. November 28 - December 01, 2017, Sanya, Hainan Island, People's Republic of China, pp. 174-179.

\title{
DETERMINATION OF THE DIMENTIONS OF STRUCTURAL ELEMENTS ON THE CONDENSED MATTER SURFACE BY WAVELET-TRANSFORMATION OF GENERATED OPTICAL IMAGES
}

\author{
(C) 2018 M. M. Filyak, A. G. Chetverikova, O. N. Kanygina \\ Orenburg State University, 13 Pobedy av., 460018 Orenburg, Russia \\ e-mail:KR-727@mail.ru
}

Received 26.01.2018

\begin{abstract}
The main tasks when it comes to the image processing of condensed matter surfaces are dispersion and morphological analyses. Determining the size and shape of the structural elements that form condensed matter is of paramount importance. Getting the most out of the image is a key issue. Recently, similar studies have been conducted using a continuous wavelet transform.

The method of determining the structural element sizes on the surface of condensed media by means of the wavelet transform of the generated models of optical images is proposed. The choice of method is due to the possibility of transition to a frequency-spatial representation of the object without preliminary image processing. A large statistical sampling and adaptation to an almost unlimited number of surface morphological features provides additional advantages.

During the simulation, the real particles of irregular shape are replaced by equivalent particles of regular geometric shapes, for example, rectangles or tetrahedra. To restore the size of real particles, the concept of an equivalent diameter is used.
\end{abstract}


Correct tetrahedrons are chosen as reference structural objects for the modelling. This choice is mainly due to the isometric or fragmented form of natural clay particles on which the method is tested.

The result of wavelet processing of optical images is the wavelet spectrum $\mathrm{W}(\mathrm{m}, \mathrm{n})$. The particle size is related to the scale $\mathrm{m}$, on which the apexes of "arches" are found by a simple relation.

The method was tested on the generated model with particle sizes $(\mu \mathrm{m})$ of 120,240 , and 600 and a natural clay powder containing fragments of fragment form $(600 \leq d \leq 630 \mu \mathrm{m})$.

The proposed method makes it possible to estimate the equivalent particle diameters values of irregular shapes in real monomorphic systems and to reveal structural inhomogeneities. This is a highly informative method and complements the method list for studying the surface structures of real materials.

Keywords: surface of condensed matter, structural element, size, image, model, wavelet spectrum.

DOI: https://doi.org/10.17308/kcmf.2018.20/487

\section{ACKNOWLEDGEMENTS}

The reported study was supported by the Russian Foundation for Basic Research and the Government of the Orenburg Region as a part of research project №17-42-560069 r_a "New optical and mathematical methods for the analysis of structural variations of disperse and nanostructured systems."

\section{REFERENCES}

1. Shkatov V. V., Shatov U. S., Scherenkova I. S. Condensed Matter and Interphases, 2013, vol. 15, no. 2, pp. 195-199. Available at: http://www.kcmf.vsu.ru/resources/t_15_2_2013_017.pdf (in Russ.)

2. Postnikov E. B., Loskutov A. Yu. Journal of Experimental and Theoretical Physics, 2007, vol. 104, no. 3. pp. 417-422. DOI: $10.1134 /$ S1063776107030065

3. Mel'nik V. M., Rud' V. D. \& Mel'nik Y. A. Powder Metallurgy and Metal Ceramics, 2014, vol. 53, no. 1-2, p. 107-112.

4. Filyak M. M., Chetverikova A. G., Kanygina O. N., Bagdasaryan L. S. Condensed Matter and Interphases, 2016, vol. 18, no. 4, pp. 578-585. Available at: http://www.kcmf. vsu.ru/resources/t 18_4_2016_016.pdf (in Russ.)

5. Kolmakov A. G., Solntsev K. A., Vityaz P. A., Il'Yushchenko A. F., Kheifets M. L., Barinov S. M. Inorganic Materials: Applied Research, 2013, vol. 4, no. 4, pp. 322-327. DOI: 10.1134/S2075113313040059

6. Mashkina E. S., Grechkina M. V. Condensed Matter and Interphases, 2013, vol. 15, no. 1, pp. 28-33. Available at: http://www.kcmf.vsu.ru/resources/t_15_1_2013_006.pdf (in Russ.)

7. Zhiznakov A. L., Fomin A. A. Fundamental Problems of Modern Materials Science, 2007, vol. 4, no. 2, pp. 75-80.
Available at: https://elibrary.ru/download/elibrary_ 12774581 90963418.pdf

8. Dobrotvorskiy S. S., Basova E. V., Repeta A. A. Bulletin of the National Technical University Kharkov Polytechnic Institute. Series: Computer Science and Modeling, 2011, no. 13, pp. 75-82.

9. Zverev V. V., Zalazinskii A. G., Novozhonov V. I., Polyakov A. P. Journal of Applied Mechanics and Technical Physics, 2001, vol. 42, no. 2, pp. 363-370. DOI: https://doi. org/10.1023/A:1018856726111

10. Machov V. E. Konstrukcii iz kompozicionnyh materialov [Composite Materials Constructions], 2010, no. 3, pp. 28-36. Available at: https://elibrary.ru/download/elibrary_15133997_95964944.pdf

11. Chetverikova A. G., Kanygina O. N., Filyak M. M., Savinkova E. S. Measurement Techniques, 2018, vol. 60, no. 11, pp. 1109-1115, DOI: 10.1007/s11018-018-1326-4

12. Hilarov V. L., Korsukov V. E., Svetlov V. N., Butenko P. N. Physics of the Solid State, 2004, vol. 46, no. 10, pp. 1868-1872. DOI: https://doi.org/10.1134/1.1809422

13. GOST R 8.755-2011 State System for Ensuring the Uniformity of Measurements. Disperse Composition of Gaseous Media. Determination of the Size of Nanoparticles by Diffusion Spectrometry. Moscow, Standardinform, 2013, $30 \mathrm{p}$.

14. Astaf'eva N. M. / Physics-Uspekhi, 1996, vol. 39, no. 11, pp. 1085-1108. DOI: http://dx.doi.org/10.1070/PU1996v039n11ABEH000177

15. Filyak M. M., Chetverikova A. G., Kanygina O. N., Savinkova E. S., Berdinskiy V. L. Proceedings of the 14th Sino-Russia Symposium on Advanced Materials and Technologies. November 28 - December 01, 2017, Sanya, Hainan Island, People's Republic of China, pp. 174-179.
Филяк Марина Михайловна - к. т. н., доцент кафедры промышленной электроники и информационно-измерительной техники, Оренбургский государственный университет; тел.: +7(3532) 372874, e-mail: filyak@mail.ru
Marina M. Filyak - Cand. Sci. (Eng.), Associate Professor of the Department of Industrial Electronics and Informing and Measuring Techniques, Orenburg State University; tel.: +7(3532) 372874, e-mail: filyak@mail.ru 
Четверикова Анна Геннадьевна - к. ф.-м. н., доцент, декан физического факультета, Оренбургский государственный университет; тел.: +7(961) 9378459, e-mail: kr-727@mail.ru

Каныгина Ольга Николаевна - д. ф.-м. н, профессор, профессор кафедры общей физики, Оренбургский государственный университет; тел. +7(3532) 372439, e-mail: onkan@mail.ru
Anna G. Chetverikova - Cand. Sci. (Phys.-Math.), Associate Professor, Dean of the Physics Department, Orenburg State University; tel.: +7(961) 9378459, e-mail: kr-727@ mail.ru

Olga N. Kanygina - Dr. Sci. (Phys.-Math.), Full Professor, Professor of the Department of General Physics, Orenburg State University, tel.: +7 (3532) 372439, e-mail: onkan@mail.ru 